\title{
HOW TO CENTRALIZE AND NORMALIZE QUANDLE EXTENSIONS
}

\author{
M. DUCKERTS-ANTOINE, V. EVEN, AND A. MONTOLI
}

\begin{abstract}
We show that quandle coverings in the sense of Eisermann form a (regular epi) reflective subcategory of the category of surjective quandle homomorphisms, both by using arguments coming from categorical Galois theory and by constructing concretely a centralization congruence. Moreover, we show that a similar result holds for normal quandle extensions.

Keywords: Quandle, quandle covering, central extension, normal extension, Galois theory.
\end{abstract}

\section{INTRODUCTION}

The origins of the structure of quandle go back to the early 40's when M. Takasaki [15] defined the notion of a kei in order to find an algebraic structure to capture the properties of reflections in a Euclidean space. A kei is defined as a set $A$ equipped with a binary operation $\triangleleft$ satisfying the following three identities for all $a, b, c \in A$

- $a \triangleleft a=a$;

- $(a \triangleleft b) \triangleleft b=a$;

- $(a \triangleleft b) \triangleleft c=(a \triangleleft c) \triangleleft(b \triangleleft c)$.

The notation $a \triangleleft b$ stands for the reflection of $a$ over $b$.

Forty years later arose the structure of quandle, as defined by D. Joyce [12]. The aim of this structure was to construe the symmetries of a geometric object on the object itself. In particular, quandles have interesting interactions with knot theory where they actually provide a knot invariant. In fact, replacing the knot group with the knot quandle, it was proved in [12] that two tame knots with isomorphic knot quandles are equivalent up to orientation.

Definition 1.1. A quandle is a set $A$ equipped with two binary operations $\triangleleft$ and $\triangleleft^{-1}$ such that for all $a, b, c \in A$ :

(Q1) $a \triangleleft a=a$

(Q2) $(a \triangleleft b) \triangleleft^{-1} b=a=\left(a \triangleleft^{-1} b\right) \triangleleft b$

(Q3) $(a \triangleleft b) \triangleleft c=(a \triangleleft c) \triangleleft(b \triangleleft c)$

(idempotency);

(right invertibility);

(self-distributivity).

Given two quandles $A$ and $B$, a function $f: A \rightarrow B$ is a quandle homomorphism when it preserves the two binary operations: the equalities $f\left(a \triangleleft a^{\prime}\right)=f(a) \triangleleft f\left(a^{\prime}\right)$ and $f\left(a \triangleleft^{-1} a^{\prime}\right)=f(a) \triangleleft^{-1} f\left(a^{\prime}\right)$ hold for all $a, a^{\prime} \in A$. We denote the category of quandles by Qnd and call a surjective quandle homomorphism an extension.

In this article, we will be mainly interested in two classes of extensions in Qnd: the classes of central and normal extensions. Both notions come from categorical Galois theory and they are defined with respect to a fundamental adjunction linking the category of quandles to its full subcategory of trivial quandles (a quandle $A$ is 
trivial when $a \triangleleft a^{\prime}=a$ and $a \triangleleft^{-1} a^{\prime}=a$ for all $a, a^{\prime}$ in $A$ ).

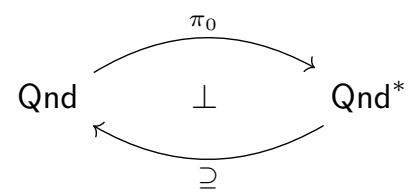

Note that the notion of central extension that we study here corresponds to another notion (specific to the context of quandles) introduced by M. Eisermann. In [9], he developed a Galois theory for what he called quandle coverings. A quandle homomorphism $f: A \rightarrow B$ is a quandle covering if it is surjective and $f(a)=f\left(a^{\prime}\right)$ implies $c \triangleleft a=c \triangleleft a^{\prime}$ for all $c \in A$. The coincidence of the two notions was proved in $[6]$.

On the contrary, normal extensions do not seem to have been studied earlier in the context of quandles. Nevertheless, they can be also described easily: a surjective homomorphism $f: A \rightarrow B$ is a normal extension if, for all $a_{i} \in A, \alpha_{i} \in\{-1,1\}$ with $0 \leq i \leq n$,

implies

$$
a_{0} \triangleleft^{\alpha_{1}} a_{1} \triangleleft^{\alpha_{2}} \cdots \triangleleft^{\alpha_{n}} a_{n}=a_{0}
$$

for all $a_{i}^{\prime} \in f^{-1}\left(f\left(a_{i}\right)\right)$.

$$
a_{0}^{\prime} \triangleleft^{\alpha_{1}} a_{1}^{\prime} \triangleleft^{\alpha_{2}} \cdots \triangleleft^{\alpha_{n}} a_{n}^{\prime}=a_{0}^{\prime}
$$

The aim of this article is to prove that it is possible to centralize and normalize any quandle extension. More formally, we shall prove that there are left adjoints to the inclusion functors $\operatorname{CExt}($ Qnd $) \hookrightarrow \operatorname{Ext}($ Qnd $)$ and $\operatorname{NExt}($ Qnd $) \hookrightarrow \operatorname{Ext}($ Qnd $)$ where CExt(Qnd) is the category of central extensions, NExt(Qnd) is the category of normal extensions, and Ext(Qnd) the category of extensions (all viewed as full subcategories of the category Qnd $^{\rightarrow}$ of arrows in Qnd). The construction of a universal central (or normal) extension associated with any surjective quandle homomorphism can be used to obtain a description of the fundamental group of a quandle, and to relate it with cohomology and extension theory of quandles. It is, moreover, the first step in order to get a homotopy theory for quandles (this is material for future work).

\section{ON THE CATEGORY OF QUANDLES}

We already recalled in the Introduction the notion of quandle. Here we provide some basic examples of quandles:

Example 2.1 ([12]). (1) Any set $A$ is a quandle with the structure defined by

$$
a \triangleleft a^{\prime}=a=a \triangleleft^{-1} a^{\prime}
$$

for all $a, a^{\prime} \in A$. Such quandles are called trivial quandles.

(2) Given a multiplicative group $G$, we can define

$$
g \triangleleft h=h^{-1} \cdot g \cdot h
$$

and

$$
g \triangleleft^{-1} h=h \cdot g \cdot h^{-1}
$$

for all $g, h \in G$. The group $G$ equipped with these operations is a quandle called the conjugation quandle. This is, in some sense, the key example of quandles. Indeed, as observed in [12], the Wirtinger presentation of the knot group of a given knot only involves conjugations. Whence the idea of replacing knot groups with knot quandles: the axioms defining a quandle have been obtained from those satisfied by the group conjugation. As we recalled in the Introduction, the knot quandle of a tame knot characterizes the knot up to orientation. 
(3) Let $n$ be a positive integer and define on $\mathbb{Z}_{n}$ the operations

$$
i \triangleleft j=2 j-i=i \triangleleft^{-1} j
$$

for all $i, j \in \mathbb{Z}_{n}$. This defines a quandle called dihedral quandle.

(4) More generally, if $G$ is a multiplicative group, we can define

$$
g \triangleleft h=h \cdot g^{-1} \cdot h=g \triangleleft^{-1} h
$$

for all $g, h \in G$. This defines a quandle called core quandle.

(5) Let $M$ be a module over the ring $\mathbb{Z}\left[t, t^{-1}\right]$ of Laurent polynomials. Define

$$
x \triangleleft y=t(x-y)+y
$$

and

$$
x \triangleleft^{-1} y=t^{-1}(x-y)+y
$$

for all $x, y \in M$. This defines a quandle called Alexander quandle. These quandles can be used to compute the Alexander polynomial of a knot.

A well-known fact about any quandle $A$ is that the axioms (Q2) and (Q3) imply that, for every $b \in A$, the right translation $(-)^{\rho_{b}}: A \rightarrow A$ defined by $a^{\rho_{b}}=a \triangleleft b$ is an automorphism.

Definition 2.2. The group $\operatorname{Inn}(A)$ of inner automorphisms of a quandle $A$ is the subgroup of $\operatorname{Aut}(A)$ (the group of all automorphisms of $A$ ) generated by all right translations $\rho_{b}$ with $b \in A$.

From a quandle $A$, we define a connected component to be an orbit under the action of the group $\operatorname{Inn}(A)$ (for $a \in A$, we write $\eta_{A}(a)$ for its orbit). The set of connected components of $A$ is denoted by $\pi_{0}(A)$ and yields a trivial quandle. The reflection of the category of quandles into its full subcategory of trivial quandles is precisely given by $\pi_{0}$ : Qnd $\rightarrow$ Qnd*, which takes a quandle $A$ and send it to its set of connected components $\pi_{0}(A)$. The component of the unit $\eta$ at $A$ is given by $\eta_{A}: A \rightarrow \pi_{0}(A): a \mapsto \eta_{A}(a)$.

We recall that a variety (in the sense of universal algebra) is a class of algebras of signature $\mathcal{F}$ satisfying a set of identities of the same signature $\mathcal{F}$, with morphisms preserving the operations in $\mathcal{F}$ (see Birkhoff's theorem [3]).

Like any other variety, Qnd is in particular a regular category [1]. This means that

(1) every morphism $f$ can be factored as $f=m \circ p$ where $m$ is a monomorphism and $p$ is a regular epimorphism (the coequaliser of some pair of parallel morphisms), and such a factorization is unique up to isomorphisms;

(2) every pullback of a regular epimorphism along any morphism is a regular epimorphism (we say that regular epimorphisms are pullback-stable).

We recall that in Qnd, as in any variety, a monomorphism is an injective homomorphism and a regular epimorphism is a surjective homomorphism. A consequence of the above axioms is that regular epimorphisms are orthogonal to monomorphisms, by which we mean that for every commutative diagram

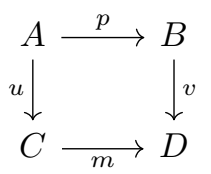

where $p$ is a regular epimorphism and $m$ a monomorphism, there exists a unique morphism $t: B \rightarrow C$ such that $u=t \circ p$ and $v=m \circ t$. In particular, this implies that a factorization $f=m \circ p$ as above is unique (up to isomorphism). We shall use this fact in Section 6 . 


\section{Central and normal extensions}

The adjunction (1) is at the heart of this paper. The reason is that it fits in the categorical Galois theory developed by G. Janelidze [10]. In particular, the categorical Galois theory studies central extensions and normal extensions defined with respect to adjunctions that satisfy a certain pullback preservation property. Such adjunctions are said to be admissible (see the beginning of Section 4 for the definition). In order to give a hint of this theory, let us restrict ourselves to the case of an adjunction

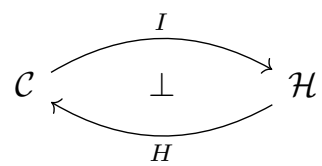

where $\mathcal{C}$ is a variety and $\mathcal{H}$ a subvariety of $\mathcal{C}$ (see [11] for a more general approach). This basically says that the components of the unit (resp. counit) are regular epimorphisms (resp. isomorphisms), $H$ is an inclusion, and $\mathcal{H}$ is closed under quotients (if $f: A \rightarrow B$ is a surjective homomorphism and $A$ is in $\mathcal{H}, B$ is also in $\mathcal{H}$ ). In particular, the adjunction is a (regular epi)-reflection.

Relatively to any adjunction (2), it is possible to define the notions of trivial extension, central extension, and normal extension. These are all special surjective homomorphisms in the category $\mathcal{C}$. A surjective homomorphism $f: A \rightarrow B$ will often be called an extension (of $B$ ).

Definition 3.1. An extension $f: A \rightarrow B$ is

- trivial when the naturality square of the adjunction

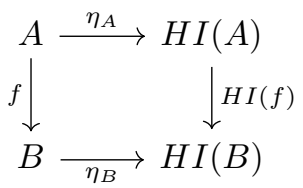

is a pullback;

- central when there exists a surjective homomorphism $p: E \rightarrow B$ such that $p_{1}: E \times{ }_{B} A \rightarrow E$ in the pullback

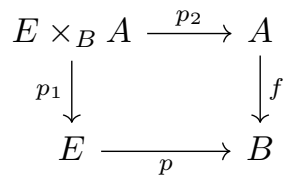

is a trivial extension (in that case we say that $f$ is split by $p$ );

- normal when $f_{1}: \operatorname{Eq}(f) \rightarrow A$ in the pullback

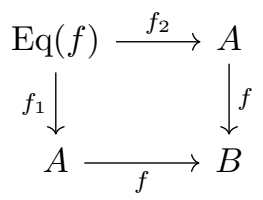

is a trivial extension (i.e. $f$ is split by itself). Here $\mathrm{Eq}(f)$ is the kernel congruence of $A$ : two elements $a_{1}, a_{2} \in A$ are in relation w.r.t. $\operatorname{Eq}(f)$ if and only if $f\left(a_{1}\right)=f\left(a_{2}\right)$.

As proved in [6], for our adjunction (1), an extension $f: A \rightarrow B$ is trivial if and only if the following condition $(T)$ holds:

$$
\forall a, a^{\prime} \in A \text {, if } f(a)=f\left(a^{\prime}\right) \text { and } \pi_{0}(a)=\pi_{0}\left(a^{\prime}\right) \text {, then } a=a^{\prime} .
$$


As explained before, the central extensions relative to the adjunction (1) turn out to be exactly the quandle coverings defined by M. Eisermann [9]: $f: A \rightarrow B$ is a quandle covering if it is surjective and $f(a)=f\left(a^{\prime}\right)$ implies $c \triangleleft a=c \triangleleft a^{\prime}$ for all $c \in A$. The proof of this result can be found in [6]. It is interesting for us to note that this proof uses the existence of a special central extension $p: \tilde{A} \rightarrow A$ for any quandle $A$ which is "weakly universal", i.e. such that every central extension of $A$ is actually split by $p$ (See [6, Theorem 2] and its proof). In particular $p: \tilde{A} \rightarrow A$ is a normal extension. We shall make use of those special normal extensions in Section 5.

In many cases, normal extensions coincide with central extensions. A typical example is given by the (regular epi)-reflection between the variety of groups and its subvariety of abelian groups

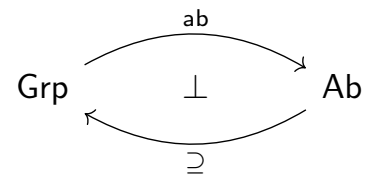

Here ab is the abelianization functor sending a group $G$ to $G /[G, G]$. The central extensions for this adjunction are exactly the classical central extensions: a group homomorphism $f: A \rightarrow B$ is central if and only if its kernel $\operatorname{Ker}(f)$ is a subgroup of the centre $Z(A)$ of $A$. For our adjunction (1), it is not true that normal and central extensions coincide. In the following proposition we give a description of the normal extensions and, via an example (see Example 3.3 below), we show that the central extensions are not always normal extensions (of course, the converse is true: every normal extension is central).

Proposition 3.2. A surjective quandle homomorphism $f: A \rightarrow B$ is a normal extension if and only if the following condition $(N)$ holds:

for all $a_{i} \in A$ (with $\left.0 \leq i \leq n\right)$ and $\alpha_{j} \in\{-1,1\}$ (with $1 \leq j \leq n$ ), if

$$
a_{0} \triangleleft^{\alpha_{1}} a_{1} \triangleleft^{\alpha_{2}} \cdots \triangleleft^{\alpha_{n}} a_{n}=a_{0}
$$

then

$$
a_{0}^{\prime} \triangleleft^{\alpha_{1}} a_{1}^{\prime} \triangleleft^{\alpha_{2}} \cdots \triangleleft^{\alpha_{n}} a_{n}^{\prime}=a_{0}^{\prime}
$$

for all $a_{i}^{\prime} \in f^{-1}\left(f\left(a_{i}\right)\right)$.

Proof. By definition, $f: A \rightarrow B$ is a normal extension if and only if the first projection $f_{1}: \operatorname{Eq}(f) \rightarrow A$ in the following diagram is a trivial extension.

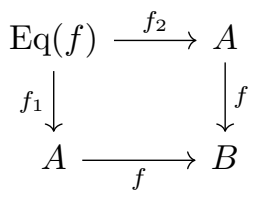

But $f_{1}: \operatorname{Eq}(f) \rightarrow A$ is a trivial extension if and only if Condition $(T)$ holds:

$\forall\left(a_{0}, a_{0}^{\prime}\right),\left(x_{0}, x_{0}^{\prime}\right) \in \operatorname{Eq}(f)$, if

$$
f_{1}\left(a_{0}, a_{0}^{\prime}\right)=f_{1}\left(x_{0}, x_{0}^{\prime}\right)
$$

(i.e. $\left.a_{0}=x_{0}\right)$ and

$$
\pi_{0}\left(\left(a_{0}, a_{0}^{\prime}\right)\right)=\pi_{0}\left(\left(x_{0}, x_{0}^{\prime}\right)\right)
$$

then

$$
\left(a_{0}, a_{0}^{\prime}\right)=\left(x_{0}, x_{0}^{\prime}\right) .
$$

This translates to the following condition: 
$\forall a_{0}, a_{0}^{\prime}, x_{0}^{\prime}$ such that $f\left(a_{0}\right)=f\left(a_{0}^{\prime}\right)=f\left(x_{0}^{\prime}\right)$, if there exists $\left(a_{i}, a_{i}^{\prime}\right) \in \operatorname{Eq}(f)$ with $1 \leq i \leq n$ such that

$$
\left(a_{0}, a_{0}^{\prime}\right) \triangleleft^{\alpha_{1}}\left(a_{1}, a_{1}^{\prime}\right) \triangleleft^{\alpha_{2}} \cdots \triangleleft^{\alpha_{n}}\left(a_{n}, a_{n}^{\prime}\right)=\left(a_{0}, x_{0}^{\prime}\right)
$$

then $\left(a_{0}, a_{0}^{\prime}\right)=\left(a_{0}, x_{0}^{\prime}\right)$, which means

$$
\left(a_{0}, a_{0}^{\prime}\right)=\left(a_{0}, a_{0}^{\prime}\right) \triangleleft^{\alpha_{1}}\left(a_{1}, a_{1}^{\prime}\right) \triangleleft^{\alpha_{2}} \cdots \triangleleft^{\alpha_{n}}\left(a_{n}, a_{n}^{\prime}\right) .
$$

Clearly, condition $(N)$ implies the previous condition, but it is also true that the previous condition implies $(N)$ since we can take $x_{0}^{\prime}$ to be

$$
x_{0}^{\prime}=a_{0}^{\prime} \triangleleft^{\alpha_{1}} a_{1}^{\prime} \triangleleft^{\alpha_{2}} \cdots \triangleleft^{\alpha_{n}} a_{n}^{\prime} .
$$

Example 3.3. Consider the involutive $\left(\triangleleft=\triangleleft^{-1}\right)$ quandle A given by

\begin{tabular}{l|llll}
$\triangleleft$ & $a$ & $b$ & $c$ & $d$ \\
\hline$a$ & $a$ & $a$ & $a$ & $a$ \\
$b$ & $b$ & $b$ & $d$ & $b$ \\
$c$ & $c$ & $c$ & $c$ & $c$ \\
$d$ & $d$ & $d$ & $b$ & $d$
\end{tabular}

and the two-elements trivial quandle $X=\{x, y\}$. Now consider $f: A \rightarrow X$ defined by $f(a)=f(b)=f(d)=x$ and $f(c)=y$. It is not a normal extension since $f(a)=f(b)$ and $a \triangleleft c=a$ but $b \triangleleft c=d \neq b$. To see that it satisfies Condition $(C)$, it suffices to observe that elements with the same image by $f$ act in the same way, when we compute $\triangleleft$ with them on the right or, in other terms, when they give the same column in the composition table above.

Let us fix some notations. It is standard to write Qnd $\rightarrow$ for the category of arrows in Qnd. The objects of Qnd $\rightarrow$ are the quandle homomorphisms and the arrows of $\mathrm{Qnd}^{\rightarrow}$ are the commutative squares in Qnd. More formally, if $f: A \rightarrow B$ and $g: C \rightarrow D$ are two objects of Qnd $\rightarrow$, a morphism from $f$ to $g$ in Qnd $\rightarrow$ is a pair $\left(\alpha_{1}: A \rightarrow C, \alpha_{0}: B \rightarrow D\right)$ such that $\alpha_{0} \circ f=g \circ \alpha_{1}$, i.e. the square

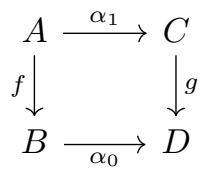

commutes.

We write $\operatorname{Ext}(\mathrm{Qnd})$ for the full subcategory of $\mathrm{Qnd}^{\rightarrow}$ whose objects are the extensions. Similarly, CExt(Qnd) (resp. NExt(Qnd)) denotes the full subcategory of $\mathrm{Qnd}^{\rightarrow}$ whose objects are central (resp. normal) extensions. The category $\operatorname{Ext}(B)$ is the full subcategory of the comma category ( $\mathrm{Qnd} \downarrow B$ ) determined by the extensions of $B$ (so that a morphism in $\operatorname{Ext}(B)$ is a commutative triangle). We will write $\operatorname{TExt}(B), \operatorname{CExt}(B)$ and $\operatorname{NExt}(B)$ for the full subcategories of $\operatorname{Ext}(B)$ determined by the trivial extensions of $B$, central extensions of $B$, and normal extensions of $B$, respectively.

The following inclusions are always true:

$$
\operatorname{TExt}(B) \subseteq \operatorname{NExt}(B) \subseteq \operatorname{CExt}(B) \subseteq \operatorname{Ext}(B) .
$$

Note that the first inclusion above comes from the admissibility of the adjunction. Indeed, for admissible adjunctions, the trivial extensions are pullback-stable [11, Proposition 4.1]. For this reason, the central and normal extensions also enjoy pullback stability [11, Proposition 4.3].

We now make some remarks on our two main problems. 
(1) To find a left adjoint to the inclusion functor CExt(Qnd) $\hookrightarrow \operatorname{Ext}($ Qnd). We are looking here for a way to transform universally any extension into a central extension. Due to the pullback stability of central extensions, we can split this problem into smaller problems: for each quandle $B$, find a left adjoint to the inclusion $\operatorname{CExt}(B) \hookrightarrow \operatorname{Ext}(B)$. In other words, if $f: A \rightarrow B$ is an extension, we are searching for a decomposition

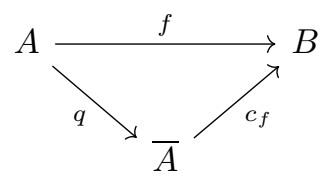

where $c_{f}$ is central and it is universal: if $f=c^{\prime} \circ q^{\prime}$ with $c^{\prime}: A^{\prime} \rightarrow B$ a central extension of $B$, there must exist a unique $\phi: \bar{A} \rightarrow A^{\prime}$ such that $c^{\prime} \circ \phi=c_{f}$ and $\phi \circ q=q^{\prime}$. We will give two methods of doing so, one of which uses a categorical approach while the other uses an algebraic approach. It turns out that $q$ is always a regular epimorphism, so that our problem further reduces to finding an appropriate congruence $R_{c}$ on $A$.

(2) To find a left adjoint to the inclusion functor NExt(Qnd) $\hookrightarrow \operatorname{Ext}($ Qnd). Although, in principle, the same procedure as above could be followed, we were not able to tackle the problem in that way. Instead, we use a general argument (the Freyd Adjoint Functor Theorem) in order to solve the problem. Note that this method can also be used to solve the first problem but its drawback is that it is not really constructive: we are left with no good information on how to construct the left adjoint we are looking for, only its existence is proved.

\section{TRIVIALizing AN EXTENSION}

The adjunction (2) is said to be admissible when the left adjoint functor $I: \mathcal{C} \rightarrow$ $\mathcal{H}$ preserves pullbacks of the following form, where $H(f)$ is a surjective homomorphism:

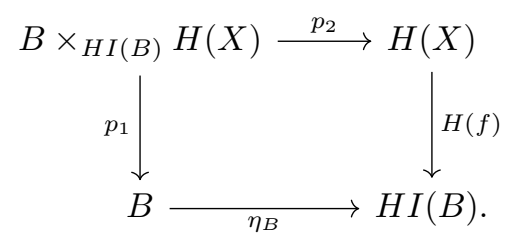

A famous example of an admissible adjunction is the reflection (6) of the category Grp into Ab. As explained in [11], this fact is a consequence of an important property of the variety Grp of groups: it is a Mal'tsev variety, i.e. a variety in which congruences over any object permute in the sense of the composition of relations. Here we adopt the classical terminology used in universal algebra and we call an equivalence relation $R \subseteq A \times A$ on (the underlying set of) a quandle A, a congruence, if it has the property that $R$ is also a subquandle of the product quandle $\left(A \times A, \triangleleft, \triangleleft^{-1}\right)$, so that, for any $(a, b) \in R$ and $\left(a^{\prime}, b^{\prime}\right) \in R$, both the elements

and

$$
(a, b) \triangleleft\left(a^{\prime}, b^{\prime}\right)=\left(a \triangleleft a^{\prime}, b \triangleleft b^{\prime}\right)
$$

belong to the relation $R$.

$$
(a, b) \triangleleft^{-1}\left(a^{\prime}, b^{\prime}\right)=\left(a \triangleleft^{-1} a^{\prime}, b \triangleleft^{-1} b^{\prime}\right)
$$

Given two congruences $R$ and $S$ on a quandle $A$, their (relational) composite $S \circ R$ is defined as the following relation on $A$ :

$$
S \circ R=\{(a, b) \in A \times A \mid \exists c \in A \text { with }(a, c) \in R \text { and }(c, b) \in S\} .
$$


For a variety $\mathcal{C}$ of algebras, having permutable congruences, meaning that we have $R \circ S=S \circ R$ for all congruences $R$ and $S$ on any object $C \in \mathcal{C}$, is equivalent to the fact that the corresponding theory has a ternary term $p(a, b, c)$ such that $p(a, b, b)=a$ and $p(a, a, b)=b$ (see [13]). For the variety of groups, such a ternary term is given by $p(a, b, c)=a \cdot b^{-1} \cdot c$. When the variety $\mathcal{C}$ is a Mal'tsev variety, the adjunction (2) is always an admissible adjunction (see [11] for a proof of this result). An interesting aspect of the adjunction (1) is that, although the variety Qnd of quandles is not a Mal'tsev variety, one can still find sufficient congruences that permute, making the adjunction (1) an admissible adjunction. These congruences were named orbit congruences in [4], where they were introduced in a completely different context.

Definition 4.1. For any subgroup $N$ of $\operatorname{Inn}(A)$, define the relation $\sim_{N} \subset A \times A$ by

$$
a \sim_{N} b \text { if and only if there exists } n \in N \text { such that } a^{n}=b .
$$

This relation is actually an equivalence relation. When moreover $N$ is a normal subgoup of $\operatorname{Inn}(A), \sim_{N}$ becomes a congruence on $A$, called an orbit congruence (see Theorem 6.1 in [4]). One can show (Lemma 2.6 in [7]) that the orbit congruences in Qnd permute with any other reflexive relation $R: \sim_{N} \circ R=R \circ \sim_{N}$. Since the kernel congruence $\operatorname{Eq}\left(\eta_{A}\right)$ of the $A$-component of the unit of the adjunction (1) $\eta_{A}: A \rightarrow \pi_{0}(A)$ is an orbit congruence (it is $\sim_{\operatorname{Inn}(A)}$ ), the adjunction (1) can be shown to be admissible for Galois theory. The proof of these facts relies on the two following results that we shall also use later on. Note that the following Lemma is a simple modification of Lemma 1.7 in [7].

Lemma 4.2. Let

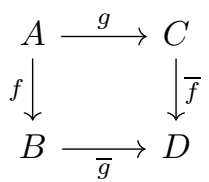

be a pushout of surjective homomorphisms in Qnd such that

$$
\operatorname{Eq}(f) \circ \operatorname{Eq}(g)=\operatorname{Eq}(g) \circ \operatorname{Eq}(f) .
$$

Then the canonical factorization $(f, g): A \rightarrow B \times{ }_{D} C$ to the pullback of $\bar{f}$ and $\bar{g}$ is a surjective homomorphism.

Corollary 4.3. [7] For any surjective homomorphism $f: A \rightarrow B$ in Qnd the commutative square

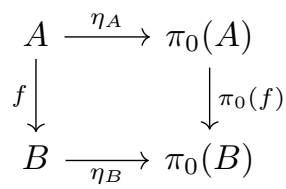

where $\eta$ is the unit of the adjunction (1) has the property that the canonical arrow $\left\langle f, \eta_{A}\right\rangle: A \rightarrow B \times_{\pi_{0}(B)} \pi_{0}(A)$ to the pullback (of $\pi_{0}(f)$ and $\eta_{B}$ ) is surjective.

Since our adjunction (1) is admissible, there exists a left adjoint to the inclusion functor $\operatorname{TExt}(B) \hookrightarrow \operatorname{Ext}(B)$ (see [11]). Actually, given a surjective quandle homomorphism $f: A \rightarrow B$, we can find a congruence $R_{t}$ on $A$ such that $t: A / R_{t} \rightarrow B$ in the factorization

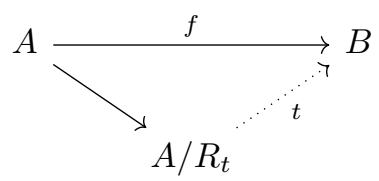


is a universal trivial extension. The trivial extension $t$ is the pullback of $\pi_{0}(f)$ along $\eta_{B}$ and $R_{t}=\mathrm{Eq}(f) \cap \sim_{\operatorname{Inn}(A)}$ is the kernel pair of the comparison morphism $\left\langle f, \eta_{A}\right\rangle: A \rightarrow B \times_{\pi_{0}(B)} \pi_{0}(A)$ so that $A \rightarrow A / R_{t}=A \rightarrow B \times_{\pi_{0}(B)} \pi_{0}(A)$ (see [7]).

\section{Centralizing an extension}

This section is devoted to the proof of our main result, namely the construction of the centralization of a quandle extension. We first describe a general construction by using categorical arguments, then we give a concrete and algebraic description of the centralization.

The first construction we consider is based on the method used by T. Everaert [8] to centralize extensions in the case of an adjunction (2) where the variety $\mathcal{C}$ is a Mal'tsev variety. We will show that the same method still works for the adjunction (1) by pointing out a weaker condition than the permutability of all congruences.

Lemma 5.1. Consider the following pullback

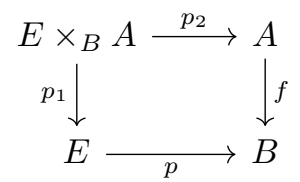

where $f: A \rightarrow B$ is a surjective quandle homomorphism and $p: E \rightarrow B$ is a normal extension. Then

$$
\operatorname{Eq}\left(p_{2}\right) \circ\left(\operatorname{Eq}\left(p_{1}\right) \cap \sim_{\operatorname{Inn}\left(E \times_{B} A\right)}\right)=\left(\operatorname{Eq}\left(p_{1}\right) \cap \sim_{\operatorname{Inn}\left(E \times_{B} A\right)}\right) \circ \operatorname{Eq}\left(p_{2}\right) .
$$

Proof. If $\left((e, a),\left(e^{\prime}, a^{\prime}\right)\right) \in \operatorname{Eq}\left(p_{2}\right) \circ\left(\operatorname{Eq}\left(p_{1}\right) \cap \sim_{\operatorname{Inn}\left(E \times_{B} A\right)}\right)$ then there exists $(\epsilon, \alpha) \in$ $E \times{ }_{B} A$ such that

$$
(e, a)\left(\operatorname{Eq}\left(p_{1}\right) \cap \sim_{\operatorname{Inn}\left(E \times_{B} A\right)}\right)(\epsilon, \alpha) \operatorname{Eq}\left(p_{2}\right)\left(e^{\prime}, a^{\prime}\right) .
$$

Thus we have $(\epsilon, \alpha)=\left(e, a^{\prime}\right)$ with $p(e)=f\left(a^{\prime}\right)$ and

$$
\pi_{0}\left(e, a^{\prime}\right)=\pi_{0}(e, a)
$$

The first condition implies that $p(e)=f\left(a^{\prime}\right)=p\left(e^{\prime}\right)$ while the second condition implies the existence of elements $\left(e_{i}, a_{i}\right) \in E \times_{B} A$ and $\alpha_{i} \in\{-1,1\}$ with $1 \leq i \leq n$ such that

$$
(e, a) \triangleleft^{\alpha_{1}}\left(e_{1}, a_{1}\right) \triangleleft^{\alpha_{2}}\left(e_{2}, a_{2}\right) \cdots \triangleleft^{\alpha_{n}}\left(e_{n}, a_{n}\right)=\left(e, a^{\prime}\right) .
$$

In particular, this shows that

and

$$
p(e)=p\left(e^{\prime}\right)
$$

$$
e \triangleleft^{\alpha_{1}} e_{1} \triangleleft^{\alpha_{2}} e_{2} \cdots \triangleleft^{\alpha_{n}} e_{n}=e .
$$

Since $p: E \rightarrow B$ is a normal extension, Proposition 3.2 implies that

$$
e^{\prime} \triangleleft^{\alpha_{1}} e_{1} \triangleleft^{\alpha_{2}} e_{2} \cdots \triangleleft^{\alpha_{n}} e_{n}=e^{\prime}
$$

Now we have that

and

$$
(e, a) \operatorname{Eq}\left(p_{2}\right)\left(e^{\prime}, a\right)
$$

which implies that

$$
\left(e^{\prime}, a\right) \triangleleft^{\alpha_{1}}\left(e_{1}, a_{1}\right) \triangleleft^{\alpha_{2}}\left(e_{2}, a_{2}\right) \cdots \triangleleft^{\alpha_{n}}\left(e_{n}, a_{n}\right)=\left(e^{\prime}, a^{\prime}\right)
$$

and thus

$$
\left(e^{\prime}, a\right)\left(\operatorname{Eq}\left(p_{1}\right) \cap \sim_{\operatorname{Inn}\left(E \times_{B} A\right)}\right)\left(e^{\prime}, a^{\prime}\right)
$$

$$
\left((e, a),\left(e^{\prime}, a^{\prime}\right)\right) \in\left(\mathrm{Eq}\left(p_{1}\right) \cap \sim_{\operatorname{Inn}\left(E \times_{B} A\right)}\right) \circ \operatorname{Eq}\left(p_{2}\right) .
$$


Note that this result does not remain true if $p$ is not asked to be a normal extension.

Example 5.2. Consider the involutive quandle A given by the following table:

\begin{tabular}{l|llll}
$\triangleleft$ & $a$ & $b$ & $c$ & $d$ \\
\hline$a$ & $a$ & $a$ & $a$ & $b$ \\
$b$ & $b$ & $b$ & $b$ & $a$ \\
$c$ & $c$ & $c$ & $c$ & $c$ \\
$d$ & $d$ & $d$ & $d$ & $d$
\end{tabular}

and $B=\{x, y\}$ the two-elements trivial quandle. Now define a quandle homomorphism $f: A \rightarrow B$ by $f(a)=f(b)=f(c)=x$ and $f(d)=y$. This quandle homomorphism has a section $s: B \rightarrow A$ (this means that $f \circ s=\operatorname{id}_{B}$ ) defined by $s(x)=c$ and $s(y)=d$. Let us consider the kernel congruence of $f$, which is given by the set $\Delta \cup\{(a, b),(a, c),(b, a),(b, c),(c, a),(c, b)\}$, where $\Delta$ is the diagonal of $A$, representing the reflexivity part of the relation: $\Delta=\{(x, x) \mid x \in A\}$.

If we compute the intersection $\operatorname{Eq}\left(p_{1}\right) \cap \sim_{\operatorname{Inn}\left(A \times{ }_{B} A\right)}$, we get

$$
\Delta \cup\{((c, a),(c, b)),((c, b),(c, a))\} .
$$

The idea is that members of $\mathrm{Eq}\left(p_{1}\right)$ share the same first element but the only couple that acts non trivially via the operation $\triangleleft i s(d, d)$ which changes the first entry of an element $(s, t)$ whenever $s=a$ or $b$. This implies that the only remaining elements are the elements from the diagonal and elements with " $c$ " in the first entry.

Now let us consider the element $((b, a),(c, b))$. This element is in

$$
\left(\operatorname{Eq}\left(p_{1}\right) \cap \sim_{\operatorname{Inn}\left(A \times{ }_{B} A\right)}\right) \circ \operatorname{Eq}\left(p_{2}\right)
$$

since

$$
(b, a) \operatorname{Eq}\left(p_{2}\right)(c, a)\left(\mathrm{Eq}\left(p_{1}\right) \cap \sim_{\operatorname{Inn}\left(A \times{ }_{B} A\right)}\right)(c, b) .
$$

But $((b, a),(c, b))$ doesn't belong to $\mathrm{Eq}\left(p_{2}\right) \circ\left(\mathrm{Eq}\left(p_{1}\right) \cap \sim_{\operatorname{Inn}\left(A \times{ }_{B} A\right)}\right)$ since

$$
(b, a)\left(\operatorname{Eq}\left(p_{1}\right) \cap \sim_{\operatorname{Inn}\left(A \times{ }_{B} A\right)}\right)(b, a)
$$

is the only choice in $\left(\mathrm{Eq}\left(p_{1}\right) \cap \sim_{\operatorname{Inn}\left(A \times_{B} A\right)}\right)$.

This permutability property is crucial for the construction, since it allows us to show that the image of a congruence remains a congruence.

Proposition 5.3. Let $f: A \rightarrow B$ be a surjective quandle homomorphism and $R$ a congruence on $A$. Then $f(R)$ is a congruence when $R \circ \mathrm{Eq}(f)=\mathrm{Eq}(f) \circ R$.

Proof. It is easy to see in general that the image of a congruence is still a reflexive and symmetric relation compatible with the operations.

Suppose that $R \circ \mathrm{Eq}(f)=\operatorname{Eq}(f) \circ R$. We have to show that $f(R)$ is a transitive relation. For this, let $\left(b_{1}, b_{2}\right)$ and $\left(b_{2}, b_{3}\right)$ be elements of $f(R)$, then there exist $\left(a_{1}, a_{2}\right) \in R$ such that $f\left(a_{1}\right)=b_{1}$ and $f\left(a_{2}\right)=b_{2}$ and $\left(a_{2}^{\prime}, a_{3}\right) \in R$ such that $f\left(a_{2}^{\prime}\right)=b_{2}$ and $f\left(a_{3}\right)=b_{3}$. In particular, we see that $\left(a_{2}, a_{2}^{\prime}\right) \in \operatorname{Eq}(f)$. It follows that $\left(a_{2}, a_{3}\right) \in R \circ \operatorname{Eq}(f)$ (since $\left.a_{2} \operatorname{Eq}(f) a_{2}^{\prime} R a_{3}\right)$ but then $\left(a_{2}, a_{3}\right) \in \operatorname{Eq}(f) \circ R$ which means that there exists $z \in A$ such that $a_{2} R z \operatorname{Eq}(f) a_{3}$. Remark that this implies that $f(z)=f\left(a_{3}\right)=b_{3}$ and thus, since $R$ is transitive, $\left(a_{1}, z\right) \in R$, showing that $\left(f\left(a_{1}\right), f(z)\right)=\left(b_{1}, b_{3}\right) \in f(R)$.

Corollary 5.4. Consider the pullback (9) where $f: A \rightarrow B$ is a surjective quandle homomorphism and $p: E \rightarrow B$ is a normal extension. Then the relation $p_{2}\left(\mathrm{Eq}\left(p_{1}\right) \cap \sim_{\operatorname{Inn}\left(E \times{ }_{B} A\right)}\right)$ is a congruence on the quandle $A$.

Proof. This follows directly from Lemma 5.1 and Proposition 5.3.

We are now in the position to prove our main theorem. 
Theorem 5.5. The category $\operatorname{CExt}(B)$ of central extensions of $B$ is a (regular epi)reflective subcategory of the category $\operatorname{Ext}(B)$.

Proof. Let $f: A \rightarrow B$ be a surjective quandle homomorphism and consider the weakly universal central extension $p: \tilde{B} \rightarrow B$, whose existence has been recalled in Section 3. Take the pullback of $f$ along $p$ :

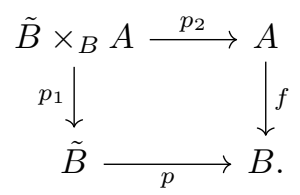

In order to simplify the notation, let us write

$$
\cap=\operatorname{Eq}\left(p_{1}\right) \cap \sim_{\operatorname{Inn}\left(\tilde{B} \times{ }_{B} A\right)} .
$$

The congruence $\cap$ is actually the congruence that trivializes $p_{1}$, i.e. such that $l:\left(\tilde{B} \times_{B} A\right) / \cap \rightarrow \tilde{B}$ is the universal trivial extension associated with $p_{1}$. Now, since $p: \tilde{B} \rightarrow B$ is a normal extension, we already know from Corollary 5.4 that $p_{2}(\cap)$ is a congruence on the quandle $A$. Now consider the following diagram

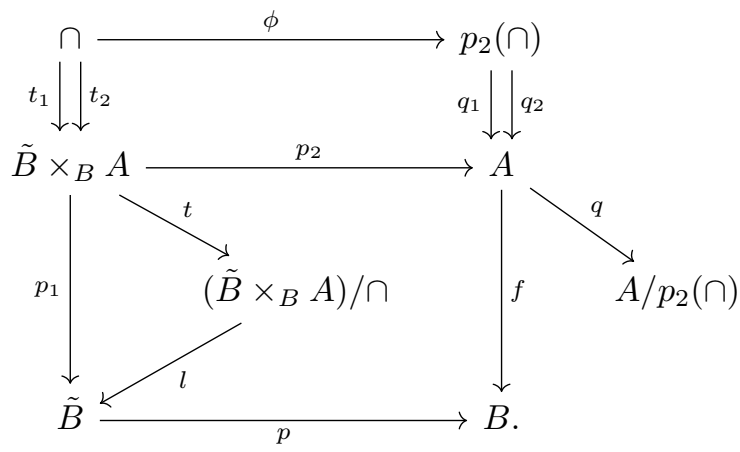

We first observe that since

$$
\begin{aligned}
q \circ p_{2} \circ t_{1} & =q \circ q_{1} \circ \phi \\
& =q \circ q_{2} \circ \phi \\
& =q \circ p_{2} \circ t_{2}
\end{aligned}
$$

there exists a unique quandle homomorphism $h:\left(\tilde{B} \times{ }_{B} A\right) / \cap \rightarrow A / p_{2}(\cap)$ such that $h \circ t=q \circ p_{2}$.

Also, since $\phi: \cap \rightarrow p_{2}(\cap)$ is a surjective quandle homomorphism and $f \circ q_{1} \circ \phi=f \circ q_{2} \circ \phi$, we have a unique quandle homomorphism $c_{f}: A / p_{2}(\cap) \rightarrow B$ such that $c_{f} \circ q=f$.

Now we observe that the square of surjective homomorphisms

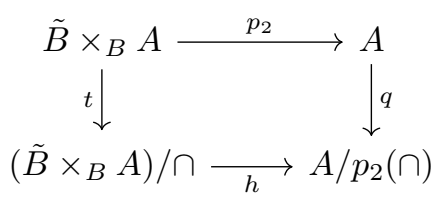

is a pushout since $\phi: \cap \rightarrow p_{2}(\cap), t: \tilde{B} \times{ }_{B} A \rightarrow\left(\tilde{B} \times{ }_{B} A\right) / \cap$ and $q: A \rightarrow A / P_{2}(\cap)$ are surjective quandle homomorphisms. By Lemma 5.1 and Lemma 4.2, this implies that the comparison morphism $\left\langle t, p_{2}\right\rangle: \tilde{B} \times_{B} A \rightarrow\left(\tilde{B} \times_{B} A\right) / \cap \times_{A / p_{2}(\cap)} A$ is surjective. $\left(p_{1}, p_{2}\right)$ being a jointly monomorphic pair, the pair $\left(t, p_{2}\right)$ is also jointly monomorphic and $\left\langle t, p_{2}\right\rangle$ is injective. Consequently, the square (10) is a pullback. 
Now we have the following situation

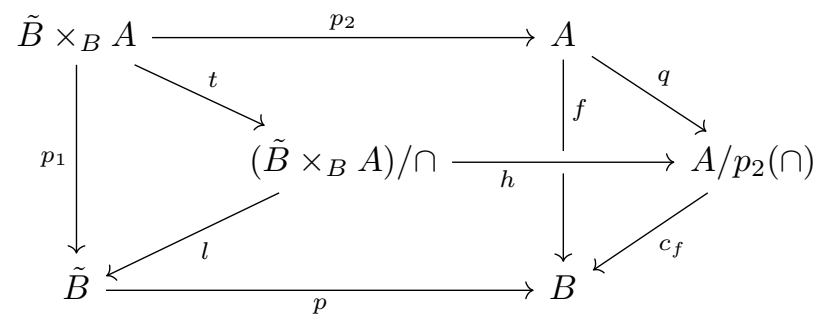

where the back square and the top square are pullbacks. Since $q: A \rightarrow A / p_{2}(\cap)$ is a surjective quandle homomorphism, the square

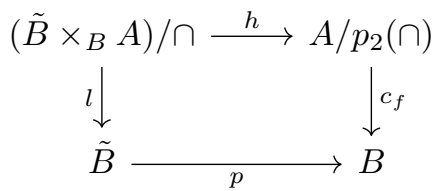

is also a pullback [11, Proposition 2.7]. Since $l$ is a trivial extension, this means that $c_{f}: A / p_{2}(\cap) \rightarrow B$ is a central extension.

All is left to show now is the universality of the construction. For this, consider the following factorization

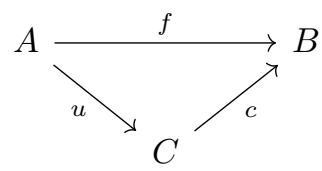

where $c: C \rightarrow B$ is a central extension. Take the pullback of $c$ along $p$

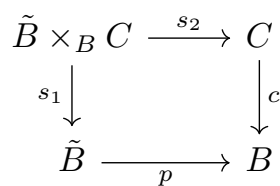

where $s_{1}: \tilde{B} \times{ }_{B} C \rightarrow \tilde{B}$ is then a trivial extension. Since the diagram

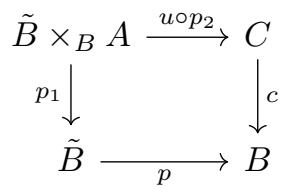

commutes, there is a unique quandle homomorphism $\beta: \tilde{B} \times{ }_{B} A \rightarrow \tilde{B} \times{ }_{B} C$ such that $u \circ p_{2}=s_{2} \circ \beta$ and $s_{1} \circ \beta=p_{1}$. By universality of the factorization $l \circ t$, there exists a unique quandle homomorphism $\gamma:\left(\tilde{B} \times{ }_{B} A\right) / \cap \rightarrow \tilde{B} \times{ }_{B} C$ such that $\beta=\gamma \circ t$ and $l=s_{1} \circ \gamma$. Thus $s_{2} \circ \gamma \circ t=s_{2} \circ \beta=u \circ p_{2}$, and since the square (10) is a pushout, this yields a unique quandle homomorphism $\alpha: A / p_{2}(\cap) \rightarrow C$ such that $\alpha \circ h=s_{2} \circ \gamma$ and $\alpha \circ q=u$. The latter equality implies that

$$
c \circ \alpha \circ q=c \circ u=f=c_{f} \circ q,
$$

and since $q$ is a surjective homomorphism, we have $c_{f}=c \circ \alpha$.

Let us give now a concrete, algebraic description of the congruence needed to produce the centralization of $f$, that is, a description of $p_{2}(\cap)$. We define explicitly 
a congruence $R_{c}$ such that $R_{c} \subseteq \mathrm{Eq}(f)$ and show directly that the induced extension $c$ in

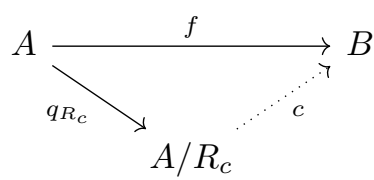

is the reflection of $f$ in $\operatorname{CExt}(B)$, so that $p_{2}(\cap)=R_{c}$. Thus, this also offers another approach to centralization. We define a relation $R$ on $A$ by putting

$$
R=\left\{\left(z \triangleleft x, z \triangleleft x^{\prime}\right) \mid z, x, x^{\prime} \in A \text { and } f(x)=f\left(x^{\prime}\right)\right\} \subseteq A \times A .
$$

This relation is reflexive and symmetric but not transitive. Moreover, it is not stable under the quandle operations, so it is not a congruence in general. We construct $R_{c}$ as the congruence generated by $R$ and we denote the corresponding quotient by $q_{R_{c}}: A \rightarrow A / R_{c}$.

The congruence $R_{c}$ is included in the kernel congruence $\operatorname{Eq}(f)$ of $f$. Indeed, every element of $R$ is included in the kernel congruence of $f$ : if $\left(z \triangleleft x, z \triangleleft x^{\prime}\right) \in R$, then

$$
f(z \triangleleft x)=f(z) \triangleleft f(x)=f(z) \triangleleft f\left(x^{\prime}\right)=f\left(z \triangleleft x^{\prime}\right) .
$$

The universal property of the quotient induces a unique quandle homomorphism $c: A / R_{c} \rightarrow B$, which is actually a central extension: if $c\left([a]_{R_{c}}\right)=c\left(\left[a^{\prime}\right]_{R_{c}}\right)$ then in particular $f(a)=f\left(a^{\prime}\right)$; this implies that $(z \triangleleft a) R_{c}\left(z \triangleleft a^{\prime}\right)$ and consequently $[z \triangleleft a]_{R_{c}}=[z \triangleleft a]_{R_{c}}$.

Now let us see that it is universal: given another factorization $f=c^{\prime} \circ q$, with $c^{\prime}: A^{\prime} \rightarrow B$ a central extension, we have to verify the existence of a unique $\phi: A / R_{c} \rightarrow A^{\prime}$ such that $c^{\prime} \circ \phi=c$ and $\phi \circ q_{R_{c}}=p$. Let $\left(z \triangleleft a, z \triangleleft a^{\prime}\right) \in R$, then $f(a)=f\left(a^{\prime}\right)$ or equivalently $c^{\prime} \circ q(a)=c^{\prime} \circ q\left(a^{\prime}\right)$. The homomorphism $c^{\prime}$ being a central extension, we have that $w \triangleleft q(a)=w \triangleleft q\left(a^{\prime}\right)$ for all $w \in A^{\prime}$. Thus by taking $w=q(z)$ we get $q(z \triangleleft a)=q\left(z \triangleleft a^{\prime}\right)$, and by the universal property of the quotient $q_{R_{c}}$ we get the desired factorization.

\section{NORMALIZING AN EXTENSION}

Now we focus our attention to the case of normal extensions. We are going to prove the following theorem.

Theorem 6.1. The category NExt(Qnd) of normal extensions is a (regular epi)reflective subcategory of the category $\operatorname{Ext}(\mathrm{Q} n \mathrm{~d})$.

For this, we first prove that the inclusion functor

$$
H_{1}: \operatorname{NExt}(\mathrm{Qnd}) \hookrightarrow \operatorname{Ext}(\mathrm{Q} \text { d) }
$$

has a left adjoint using the Freyd Adjoint Functor Theorem (see, for example, Theorem 9.9 in [2]):

Theorem 6.2. Given a small-complete category $\mathcal{A}$ with small hom-sets, a functor $G: \mathcal{A} \rightarrow \mathcal{X}$ has a left adjoint if and only if it preserves all small limits and satisfies the so-called Solution Set Condition: for each object $X \in \mathcal{X}$ there is a set $\mathcal{S}_{X}$ of objects of the comma category $(X \downarrow G)$ such that for every object $Y$ of $(X \downarrow G)$ there is a morphism $S \rightarrow Y$ in $(X \downarrow G)$ with $S$ in $\mathcal{S}_{X}$.

Recall that an object of $(X \downarrow G)$ is an arrow in $\mathcal{X}$ of the form $f: X \rightarrow G(A)$ for some $A$, and a morphism in $(X \downarrow G)$ from $f: X \rightarrow G(A)$ to $f^{\prime}: X \rightarrow G\left(A^{\prime}\right)$ is an arrow $a: A \rightarrow A^{\prime}$ in $\mathcal{A}$ such that $f^{\prime}=G(a) \circ f$.

We split the proof into several lemmas.

Lemma 6.3. The category $\operatorname{Ext}(\mathrm{Qnd})$ is small-complete. 
Proof. Let us first recall that all small limits exist if small products and equalizers exist. First, all small products in Ext(Qnd) exist and are computed as in Qnd $\rightarrow$, i.e. the product of a family $\left(f_{i}: A_{i} \rightarrow B_{i}\right)_{i \in I}$ of surjective quandle homomorphisms is given by $\prod_{i \in I} f_{i}: \prod_{i \in I} A_{i} \rightarrow \prod_{i \in I} B_{i}$. Now, let $\alpha=\left(\alpha_{1}, \alpha_{0}\right): f \rightarrow g$ and $\beta=\left(\beta_{1}, \beta_{0}\right): f \rightarrow g$ be two parallel morphisms in Ext(Qnd). The equalizer of $(\alpha, \beta)$ is given by $\left(\left(e_{1}, e_{0} \circ m\right): p \rightarrow f\right)$ where $\left(\left(e_{1}, e_{0}\right): e \rightarrow f\right)$ is the equaliser of $(\alpha, \beta)$ in $\mathrm{Qnd}^{\rightarrow}$ and $p$ and $m$ come from the regular epi-mono factorization $e=m \circ p$ :

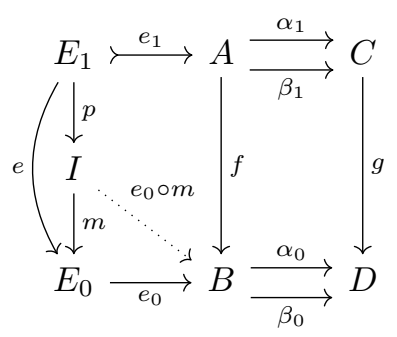

Lemma 6.4. NExt(Qnd) is closed under subobjects in $\operatorname{Ext}(\mathrm{Qnd})$.

Proof. A morphism $\left(\alpha_{1}, \alpha_{0}\right)$ in $\operatorname{Ext}($ Qnd)

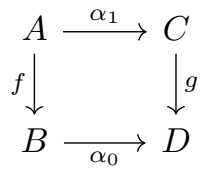

is a monomorphism if and only if $\alpha_{1}$ is an injective homomorphism. Indeed, suppose that $\alpha_{1}$ is injective and consider the diagram:

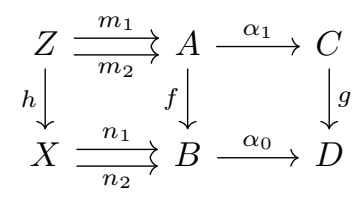

where $\alpha_{1} \circ m_{1}=\alpha_{1} \circ m_{2}$ and $\alpha_{0} \circ n_{1}=\alpha_{0} \circ n_{2}$. Since $\alpha_{1}$ is injective, we get that $m_{1}=m_{2}$ and from the surjectivity of $h$ we obtain that $n_{1}=n_{2}$. Conversely, suppose that $\left(\alpha_{1}, \alpha_{0}\right)$ is a monomorphism in $\operatorname{Ext}($ Qnd) and consider two quandle homomorphisms $m_{1}, m_{2}: Z \rightarrow A$ such that $\alpha_{1} \circ m_{1}=\alpha_{1} \circ m_{2}$. Then we can consider the following commutative diagram:

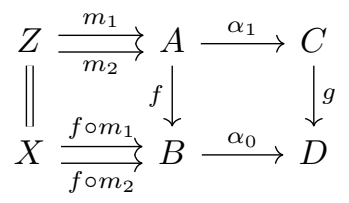

Being $\left(\alpha_{1}, \alpha_{0}\right)$ a monomorphism in $\operatorname{Ext}($ Qnd $)$, we get that $m_{1}=m_{2}$.

We want to prove that if $g$ is a normal extension and $\alpha_{1}$ an injective homomorphism, $f$ is a normal extension, too.

Let $a_{i}, a_{i}^{\prime}$ be elements of $A$ and $\alpha_{i}$ be elements of $\mathbb{Z}$ for $0 \leq i \leq n$ such that

$$
a_{0} \triangleleft^{\alpha_{1}} a_{1} \triangleleft^{\alpha_{2}} \cdots \triangleleft^{\alpha_{n}} a_{n}=a_{0}
$$

and $f\left(a_{i}\right)=f\left(a_{i}^{\prime}\right)$. We must show that

$$
a_{0}^{\prime} \triangleleft^{\alpha_{1}} a_{1}^{\prime} \triangleleft^{\alpha_{2}} \cdots \triangleleft^{\alpha_{n}} a_{n}^{\prime}=a_{0}^{\prime} .
$$

For this, it suffices to show that

$$
\alpha_{1}\left(a_{0}^{\prime}\right) \triangleleft^{\alpha_{1}} \alpha_{1}\left(a_{1}^{\prime}\right) \triangleleft^{\alpha_{2}} \cdots \triangleleft^{\alpha_{n}} \alpha_{1}\left(a_{n}^{\prime}\right)=\alpha_{1}\left(a_{0}^{\prime}\right)
$$


since $\alpha_{1}$ is injective. But we know that

$$
\alpha_{1}\left(a_{0}\right) \triangleleft^{\alpha_{1}} \alpha_{1}\left(a_{1}\right) \triangleleft^{\alpha_{2}} \cdots \triangleleft^{\alpha_{n}} \alpha_{1}\left(a_{n}\right)=\alpha_{1}\left(a_{0}\right)
$$

and $g\left(\alpha_{1}\left(a_{i}\right)\right)=\alpha_{0}\left(f\left(a_{i}\right)\right)=\alpha_{0}\left(f\left(a_{i}^{\prime}\right)\right)=g\left(\alpha_{1}\left(a_{i}^{\prime}\right)\right)$. The result follows by normality of $g$.

Lemma 6.5. NExt(Qnd) is small-complete and $H_{1}$ preserves small limits.

Proof. It is easy to show that the subcategory NExt(Qnd) is closed under small products computed in $\operatorname{Ext}(\mathrm{Q} n)$. The fact that it is also closed under equalizers follows from the fact that it is closed under subobjects. Consequently, NExt(Qnd) is closed under all small-limits in $\operatorname{Ext}($ Qnd) and the result follows.

Lemma 6.6. The inclusion functor $H_{1}$ satisfies the Solution Set Condition.

Proof. For any quandle $A$, we define

$$
Q_{A}=\{\text { canonical projection } \pi: A \rightarrow A / R \mid R \text { congruence on } A\}
$$

to be the set of canonical representatives of quotients of $A$ (there is only a set of congruences on $A$ ). As in every variety, any surjective quandle homomorphism $q: A \rightarrow Q$ is isomorphic to an element $\pi_{q}: A \rightarrow A / \operatorname{Eq}(q)$ of $Q_{A}$, i.e. $q=i_{q} \circ \pi_{q}$ for a (unique) isomorphism $i_{q}$ :

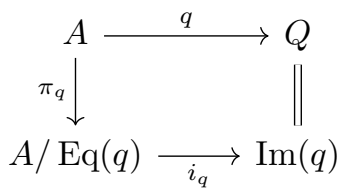

Let $f: A \rightarrow B$ be an object of $\operatorname{Ext}($ Qnd). Consider the class of pairs

$$
(q: A \rightarrow Q, g: Q \rightarrow B)
$$

with $q$ a surjective homomorphism and $g$ a normal extension such that $f=g \circ q$. Since $q$ is an epimorphism, such a $g$ is unique, hence the existence of $g$ can be viewed as a property $\varphi(q)$ of the morphism $q$ :

$$
\varphi(q) \equiv \text { there exists } g \text { normal such that } f=g \circ q .
$$

It is obvious that $\varphi(q)$ holds if and only if $\varphi\left(\pi_{q}\right)$ holds. We define $\mathcal{S}_{f}$ to be the set

$$
S_{f}=\left\{\left(\pi, 1_{B}\right): f \rightarrow H_{1}(g) \mid \pi \text { is in } Q_{A}\right\}
$$

of objects of $\left(f \downarrow H_{1}\right)$ and $\overline{\mathcal{S}}_{f}$ to be the class of objects of $\left(f \downarrow H_{1}\right)$ of the form

$$
\left(q, 1_{B}\right): f \rightarrow H_{1}(g)
$$

where $q$ is a surjective homomorphism (and $g$ is normal). For any object $y=$ $\left(q, 1_{B}\right): f \rightarrow H_{1}(g)$ of $\overline{\mathcal{S}}_{f}$, there is a morphism from the object

$$
s_{y}=\left(\pi_{q}, 1_{B}\right): f \rightarrow H_{1}\left(g \circ i_{q}\right)
$$

in $\mathcal{S}_{f}$ to $y$ given by

$$
\left(i_{q}, 1_{B}\right): g \circ i_{q} \rightarrow g
$$

Now let $y=\left(\alpha_{1}, \alpha_{0}\right): f \rightarrow H_{1}(g)$ be an object of $\left(f \downarrow H_{1}\right)$. Consider the commutative diagram in $\mathrm{Qnd}$

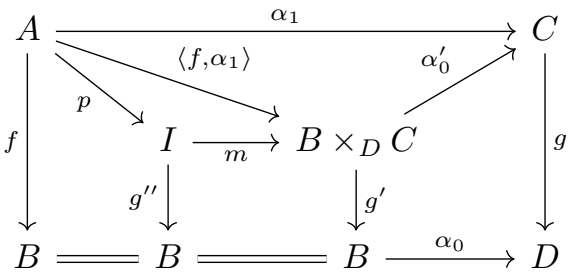


where

- $\left(B \times{ }_{D} C, g^{\prime}, \alpha_{0}^{\prime}\right)$ is a pullback of $\alpha_{0}$ and $g$;

- $\left\langle f, \alpha_{1}\right\rangle$ is the unique induced morphism such that $f=g^{\prime} \circ\left\langle f, \alpha_{1}\right\rangle$ and $\alpha_{1}=\alpha_{0}^{\prime} \circ\left\langle f, \alpha_{1}\right\rangle$

- $\left\langle f, \alpha_{1}\right\rangle=m \circ p$ is a (regular epi)-mono factorization of $\left\langle f, \alpha_{1}\right\rangle$;

- $g^{\prime \prime}=g^{\prime} \circ m$.

Since $f$ is surjective, $g^{\prime \prime}$ is a surjective homomorphism, too. Therefore, it is also a subobject in $\operatorname{Ext}\left(\mathrm{Q}\right.$ d) of the normal extension $g^{\prime}$ and, consequently, a normal extension. We define $y^{\prime}$ to be the object of $\overline{\mathcal{S}}_{f}$

$$
y^{\prime}=\left(p, 1_{B}\right): f \rightarrow H_{1}\left(g^{\prime \prime}\right)
$$

and we see that there is an arrow from $y^{\prime}$ to $y$, namely

$$
\left(\alpha_{0}^{\prime} \circ m, \alpha_{0}\right): g^{\prime \prime} \rightarrow g .
$$

Combining this with the previous observations, we find at least one object $s=s_{y^{\prime}}$ in the set $\mathcal{S}_{f}$ and an arrow from $s$ to $y$. This concludes the proof.

We have just proved that a left adjoint to $H_{1}$ exists. It remains to prove that every component of the unit of the adjunction is a regular epimorphism. This comes from the following observations:

(1) It is easy to show that, for an object $s$ in $S_{f}$ (or $\left.\bar{S}_{f}\right)$ and $y$ in $\left(f \downarrow H_{1}\right)$, there is at most one arrow $s \rightarrow y$ in the category $\left(f \downarrow H_{1}\right)$.

(2) Consequently, the initial object of $\left(f \downarrow H_{1}\right)$ (i.e. the reflection of $f$ in $\operatorname{NExt}\left(\mathrm{Q}\right.$ d)) can be chosen in $S_{f}$. Indeed, it is direct to check that, for $\bar{y}$ the reflection of $f$ in $\operatorname{NExt}(\mathrm{Q} n \mathrm{n}), s_{\bar{y}^{\prime}}$ is also initial, so that $s_{\bar{y}^{\prime}} \cong \bar{y}$. This means that the reflection of $f$ in $\operatorname{NExt}(Q n d)$ is, up to isomorphisms, of the form $\eta_{f}^{1}=\left(\pi, 1_{B}\right): f \rightarrow H_{1}\left(n_{f}\right)$ for some $\pi$ in $Q_{A}$ (we denote by $n_{f}$ the normalization of $f)$. If we write $\left(R_{n}, \pi_{1}, \pi_{2}\right)$ for the congruence associated with $\pi$, we find that

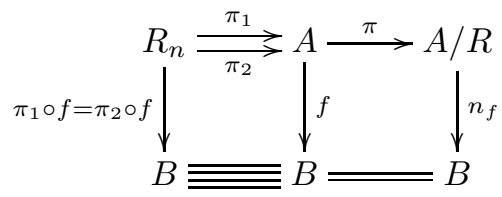

is a coequalizer diagram in $\operatorname{Ext}(\mathrm{Qnd})$. This conclude the proof of Theorem 6.1 .

Note that we can prove in a very similar way that the inclusion CExt(Qnd) $\hookrightarrow$ Ext(Qnd) has a left adjoint. Indeed, the category CExt(Qnd) enjoy the same closure properties as NExt(Qnd) inside Ext(Qnd).

More generally, the same kind of proof (with some obvious modifications) can be used to obtain the following general result:

Theorem 6.7. Let

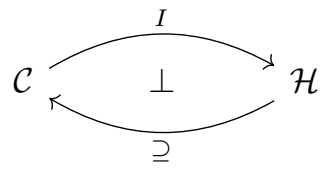

be an admissible adjunction, where $\mathcal{C}$ is a variety and $\mathcal{H}$ is a subvariety of $\mathcal{C}$. Let us assume that $\operatorname{NExt}(\mathcal{C})$ (resp. $\operatorname{CExt}(\mathcal{C})$ ) is closed under subobjects and small products in $\operatorname{Ext}(\mathcal{C})$. Then $\operatorname{NExt}(\mathcal{C})($ resp. $\operatorname{CExt}(\mathcal{C}))$ is a (regular epi)-reflective subcategory of $\operatorname{Ext}(\mathcal{C})$. 
Contrary to the case of central extensions, for normal extensions we haven't been able to find a concrete description of the congruence $R_{n}$ whose quotient gives the normalization of a quandle extension, since the Adjoint Functor Theorem is not really constructive. A description of such a congruence (whose existence is a consequence of Theorem 6.1) is an open problem.

\section{ACKNOWLEDGEMENTS}

This work was partially supported by the FCT - Fundação para a Ciência e a Tecnologia - under the grant number SFRH/BPD/98155/2013, and by the Centre for Mathematics of the University of Coimbra - UID/MAT/00324/2013, funded by the Portuguese Government through FCT/MEC and co-funded by the European Regional Development Fund through the Partnership Agreement PT2020.

This work was partially supported by a FNRS grant Crédit pour bref séjour à l'étranger that has allowed the second author to visit the Universidade de Coimbra.

This work was partially supported by the Programma per Giovani Ricercatori "Rita Levi-Montalcini", funded by the Italian government through MIUR.

\section{REFERENCES}

[1] M. Barr, Exact categories, in: Exact categories and categories of sheaves, Vol. 236 of Lecture Notes in Math., Springer, 1971, pp. 1-120.

[2] M. Barr and C. Wells, Toposes, triples and theories, Springer-Verlag, 1985.

[3] G. Birkhoff, On the structure of abstract algebras, Proc. Camb. Philos. Soc. 31 (1935), 433454.

[4] E. Bunch, P. Lofgren, A. Rapp and D. N. Yetter, On quotients of quandles, J. Knot Theory Ramifications 19, no. 9 (2010), 1145-1156.

[5] A. Carboni, G. M. Kelly and M. C. Pedicchio, Some remarks on Maltsev and Goursat categories, Appl. Categ. Struct. 1 (1993), 385-421.

[6] V. Even, A Galois-theoretic approach to the covering theory of quandles, Appl. Categ. Structures 22, no. 5-6 (2014), 817-831.

[7] V. Even and M. Gran, On factorization systems for surjective quandle homomorphisms, J. Knot Theory Ramifications 23, no. 11 (2014), 1450060.

[8] T. Everaert, Higher central extensions in Mal'tsev categories, Appl. Categ. Structures 22 (2014), 961-979.

[9] M. Eisermann, Quandle coverings and their Galois correspondence, Fund. Math. 225, no. 1 (2014), 103-168.

[10] G. Janelidze, Pure Galois Theory in Categories, J. Algebra 132 (1990), 270-286.

[11] G. Janelidze and G. M. Kelly, Galois theory and a general notion of central extension, J. Pure Appl. Alg. 97 (1994), 135-161.

[12] D. Joyce, A classifying invariant of knots, the knot quandle, J. Pure Appl. Alg. 23 (1982), $37-65$.

[13] A. I. Mal'cev, On the general theory of the algebraic systems, Mat. Sbornik (N.S.) 35 (1954), 3-20.

[14] S. V. Matveev, Distributive groupoids in knot theory, Math. Sb. (N.S.) 119(161), no. 1(9) (1982),78-88.

[15] M. Takasaki, Abstractions of symmetric transformations, Tôhoku Math. J. 49 (1943), 143207.

Centre for Mathematics, University of Coimbra, Department of Mathematics, Apartado 3008, 3001-501 Coimbra, Portugal

E-mail address: mathieud@mat.uc.pt

Institut de Recherche en Mathématique et Physique, Université Catholique de Louvain, Chemin du Cyclotron 2, 1348 Louvain-la-Neuve, Belgium.

E-mail address: valerian.even@uclouvain.be

Dipartimento di Matematica "Federigo Enriques", Università degli Studi di Milano, Via Saldini 50, 20133 Milano, Italy.

E-mail address: andrea.montoli@unimi.it 